\title{
On two generalizations of the final value theorem: scientific relevance, first applications, and physical foundations
}

\author{
Emanuel Gluskin* (EE Dept., HIT, gluskin@ee.bgu.ac.il) and Joris Walraevens \\ (Department of Telecommunications and Information Processing, Ghent University, \\ jw@telin.UGent.be)
}

\begin{abstract}
The present work considers two published generalizations of the Laplacetransform final value theorem (FVT), and some recently appeared applications of one of these generalizations to the fields of physical stochastic processes and Internet queueing. Physical sense of the irrational time functions, involved in the other generalization, is one of the points of concern. The work strongly extends the conceptual frame of the references and outlines some new research directions for applications of the generalized theorem.
\end{abstract}

Index terms: Final value theorem, Control theory, Irrational functions, Noninteger integration, Queueing theory, Ergodicity. 


\section{Introduction}

The present work is motivated, on one side, by the appearance of the applications $[1,2]$ and [3-5] of the generalization [6] of the Laplace transform final value theorem (FVT) [6-9], and, on the other side, by the more recent work [10] devoted to another generalization of the classical FVT. We discuss applications of both generalizations and explore the use of irrational functions in the last generalization. Since such functions are rare in system theory, it was found necessary to go deeper into their physical origination. This leads to some interesting analogies, which can encourage one to use such functions. These topics should be interesting to system specialists.

The work is composed of three parts. The first part (section 2) describes some classical system situations to which the generalized FVT (GFVT) should be applied. The second part (section 3) is devoted to application of irrational functions to some control and signal processing problems. Finally, the third part (section 4) is devoted to modern applications of the GFVT. The connections between the topics involved are summarized in Fig. 1. Since some connections are best/naturally seen via physics, others via mathematics, our argumentation is both physical and mathematical.

The generalizations of [6] and [10] are very different, and for clarity, the notation GFVT is usually used only for the generalization of [6], as shown in Fig. 1. This in no sense decreases the importance of the suggestions of [10], and in fact, the discussion of irrational functions influences the discussion of control systems (in section 2) and gives some support to the discussion of queueing theory (section 4) where sequences with non-integer powers arise. 


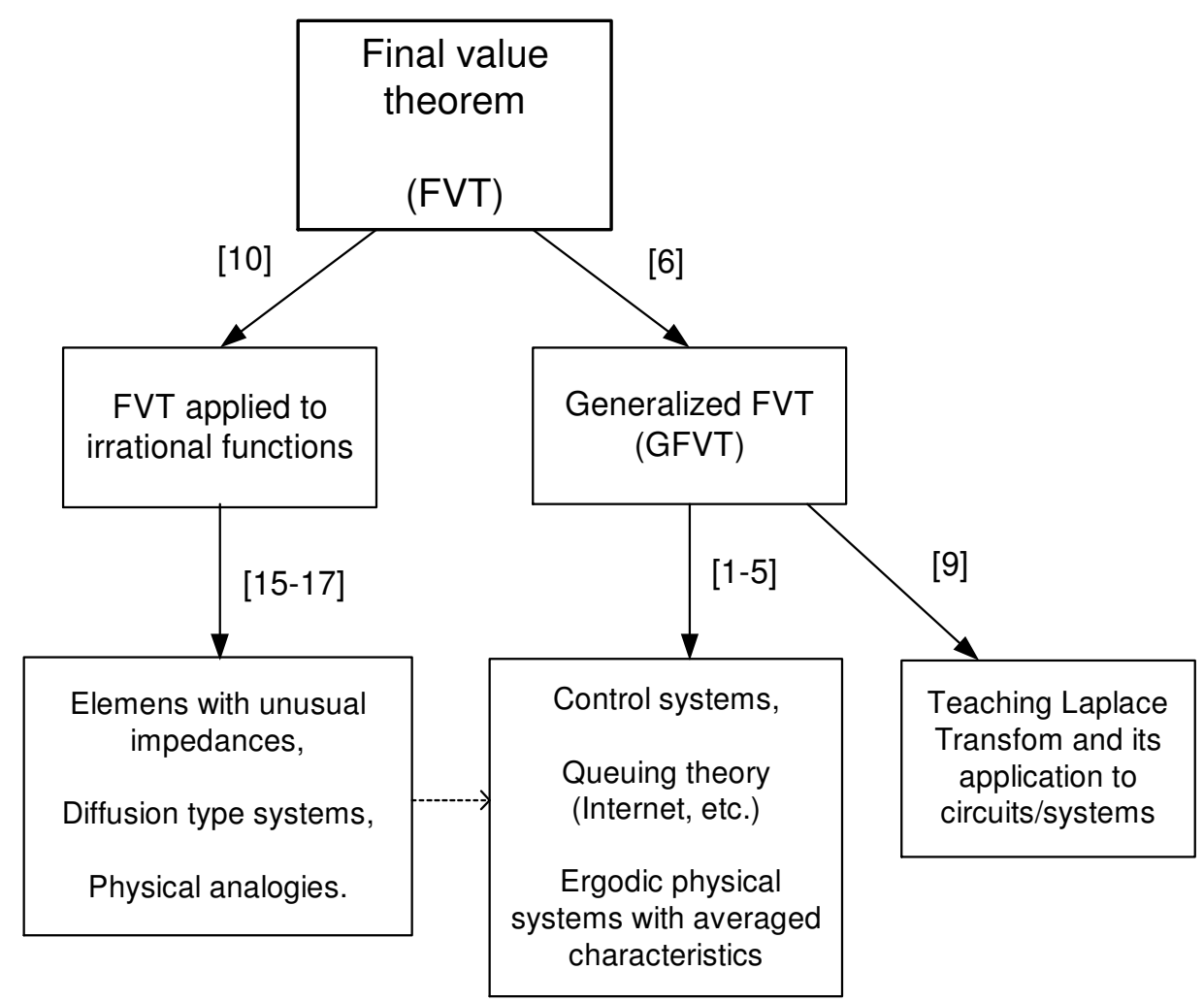

Fig. 1: The conceptual frame.

\section{Application of GFVT to classical systems}

\subsection{The classical final value theorem}

Consider the one-directional Laplace transform $F(s)$ of $f(t)$,

$$
F(s)=\int_{0}^{\infty} e^{-s t} f(t) d t
$$

The classical final value theorem states that

$$
\lim _{s \rightarrow 0} s F(s)=f(\infty) .
$$


When $f(t)$ arises as a solution function, depending on the structure of a physical or engineering system and its given input, equation (1) becomes relevant to the dynamics of the system. Though function $f(t)$ can arise in different systems, linear or nonlinear, Laplace transforms are especially effective for linear ones. Therefore, we consider only linear equations, both ordinary and in partial derivatives.

\subsection{Possible nonexistence of $f(\infty)$}

The classical FVT requires existence of $f(\infty)$. Since in physical reality this is not always known beforehand, the case when $f(\infty)$ does (potentially) not exist is most interesting to study.

There are two main possibilities for non-existence of $f(\infty)$ : the suggestion of [10] to generalize (1) to include the case when $f(t) \rightarrow \infty$ as $t \rightarrow \infty$ (and, respectively, $s \rightarrow 0$ in the $s$-domain), and the suggestion of the earlier work [6] to use the time average over the whole interval $(0, \infty)$ instead of the final value $f(\infty)$.

The tendency to infinity in [10] is understood as, for instance the tendency of $e^{t}$, or $t e^{t}$, but not of $e^{t} \sin t$. Therefore, if we use the one-to-one map of $f$ on $\varphi$ given by the equality $\operatorname{tg} \frac{\pi \varphi}{2}=f$, then $f(t) \rightarrow \infty$ is turned into $\varphi(t) \rightarrow 1$, and $f(t) \rightarrow-\infty$ into $\varphi(t) \rightarrow-1$. The "infinity" can thus be transformed into a finite value.

As for the suggestion of [6], defining the time-average over finite interval as

$$
\langle f(t)\rangle_{t} \equiv \frac{1}{t} \int_{0}^{t} f(\mu) d \mu
$$

we have the time-average of interest as the limit for $t \rightarrow \infty$ :

$$
\langle f(t)\rangle \equiv \lim _{t \rightarrow \infty}\langle f(t)\rangle_{t}=\lim _{t \rightarrow \infty} \frac{1}{t} \int_{0}^{t} f(\mu) d \mu
$$

We thus replace (1) by 


$$
\lim _{s \rightarrow 0} s F(s)=\langle f(t)\rangle
$$

which replaces the requirement, in FVT, of existence of $f(\infty)$ by the much weaker requirement of a boundedness of $f(t)$, providing existence of $\langle f(t)\rangle$.

Clearly, (2) is a generalization of (1); if $f(\infty)$ exists, $\langle f(t)\rangle=f(\infty)$. Indeed, since in this case $f(t)$ is almost all the time close to $f(\infty)$, it is clear that

$$
\lim _{t \rightarrow \infty} \frac{1}{t} \int_{0}^{t} f(\mu) d \mu=f(\infty)
$$

The proof of (2), for the cases when $f(\infty)$ does not exist, is given in section 4 .

\subsection{Comments on (1) and (2)}

1. The sinusoidal $f(t)$ is the simplest example [8,9] warning against careless use of (1), since for this function $\lim s F(s)=0$, but $f(\infty)$ does not exist. It is also $s \rightarrow 0$

the simplest supporting example for (2) since in this case $\lim _{s \rightarrow 0} s F(s)$ equals the zero time average of the sinusoid.

2. It needs to be stressed that the values of $f(\infty)$ and of $\langle f(t)\rangle$ are influenced only by the asymptotic behavior of $f(t)$ as $t \rightarrow \infty$, and thus $f(t)$ may here denote any function having the same asymptotic behavior. This observation is important when approximations of the functions of interest are needed.

3. We generally require that $\lim s F(s)$ exists, and it is argued (section 4.1) $s \rightarrow 0$

that at least for a function that possesses a simple spectral presentation, $\lim _{s \rightarrow 0} s F(s)$ and $\langle f(t)\rangle$ exist simultaneously.

4. It is worth stressing that $\langle f(t)\rangle$ does not exist for $e^{t} \sin t$ and also for $t^{\alpha} \sin t$ for $\alpha \geq 1$. The latter is seen from the equality 


$$
<f>_{t}=\frac{1}{t} \int_{0}^{t} \mu^{\alpha} \sin \mu d \mu=-t^{\alpha-1} \cos t+\alpha \frac{1}{t} \int_{0}^{t} \mu^{\alpha-1} \cos \mu d \mu
$$

obtained using integration by parts, which is correct for any $\alpha>0$. The oscillations in $\langle f\rangle_{t}$ do not vanish for $\alpha \geq 1$ as $t \rightarrow \infty$.

5. The practical use of average is associated, in particular, with the fact that any averaging means some low-pass filtering. This use of averaging is standard in signal processing [11], and is expected to be of interest for control problems where high-frequency noise, influencing the system input and its structure, has to be filtered (averaged). The topic is also relevant to spatial filtering structures where the input and output variables are spatially distributed; see [12,13] and references there.

\subsection{The averaging of vectors}

Consider the vector-function, denoted as $\mathbf{x}(t)$ or as $\vec{x}(t)$,

$$
\vec{x}(t)=\left(x_{1}(t), x_{2}(t), \ldots, x_{n}(t)\right)^{T},
$$

for which we can consider the limiting value $\vec{x}(\infty)=\left(x_{1}(\infty), x_{2}(\infty), \ldots, x_{n}(\infty)\right)^{T}$.

We define the averaged vector

$$
<\vec{x}(t)>=<\left(x_{1}(t), x_{2}(t), \ldots, x_{n}(t)\right)^{T}>=\left(<x_{1}(t)>,<x_{2}(t)>, \ldots,<x_{n}(t)>\right)^{T},
$$

assuming that all of the averaged components exist.

Then, for a constant matrix [A] of proper size we have

$$
<[A] \vec{x}(t)>=[A]<\vec{x}(t)>
$$

which, for $\vec{x}(\infty)$ existing, becomes, as $t \rightarrow \infty$, the identity $[A] \vec{x}(\infty)=[A] \vec{x}(\infty)$.

Passing now on to time-dependent matrices relevant to linear time variant (LTV) systems, we can compare

$$
\lim _{t \rightarrow \infty}([A(t)] \vec{x}(t))
$$

with

$$
<[A(t)] \vec{x}(t)>
$$


Considering each component of vector $[A(t)] \vec{x}(t)$ in view of (3) (as "f(t)"), and then applying (4) to the whole vector, we see that if the limit-vector (5) exists, then average (6) equals (5).

\subsection{The use of the transfer matrix}

Consider now the transfer matrix function $[H(s)]$ of a general LTI system. We are interested in the time averages of the input function $\vec{u}(t)$ and output function $\vec{y}(t)$.

By definition of $[H(s)]$,

$$
\vec{Y}(s)=[H(s)] \vec{U}(s),
$$

with $\vec{Y}(s)$ and $\vec{U}(s)$ Laplace transforms of $\vec{y}(t)$ and $\vec{u}(t)$. In view of GFVT (2), the time average of the $\vec{u}(t)$ simply results in the time average of $\vec{y}(t)$ :

$$
\begin{aligned}
& \langle\vec{y}(t)\rangle=\lim _{s \rightarrow 0}(s \vec{Y}(s))=\lim _{s \rightarrow 0}(s[H(s)] \vec{U}(s)) \\
& =[H(0)] \lim _{s \rightarrow 0}(s \vec{U}(s))=[H(0)]\langle\vec{u}(t)\rangle
\end{aligned} .
$$

Since $[H(0)]$ is physically realized by ignoring all of the differential elements having memory, this matrix is real-valued, and, as such, can be passed on to the time domain.

Equality (7) includes the case $\vec{y}(\infty)=[H(0)] \vec{u}(\infty)$. For existence of $\vec{y}(\infty)$, it is sufficient to require stability of the system and existence of $\vec{u}(\infty)$.

Let us determine $[H(0)]$ for the case of the finite values existing. Consider the LTI state equations

$$
\begin{aligned}
& \frac{d \vec{x}}{d t}=[A] \vec{x}+[B] \vec{u} \\
& \vec{y}=[C] \vec{x}+[D] \vec{u}
\end{aligned}
$$

with a nonsingular matrix [A]. Assuming that $\vec{u}(\infty), \vec{x}(\infty)$ and $\vec{y}(\infty)$ exist, we have for $t \rightarrow \infty$, that $\frac{d \vec{x}}{d t} \rightarrow \overrightarrow{0}$ (the system becomes algebraic), and (8) leads to

$$
\vec{y}(\infty)=\left([D]-[C][A]^{-1}[B]\right) \vec{u}(\infty)=[H(0)] \vec{u}(\infty),
$$


that is,

$$
[H(0)]=[D]-[C][A]^{-1}[B]
$$

This can also be obtained by setting $s=0$ in the general transfer matrix of the system

$$
[H(s)]=[C][s I-A]^{-1}[B]+[D] .
$$

In the context of the averages, the substitution $s=0$ has a deeper meaning than it is seen at first. Namely, we argue below (Section 3) that, for physical processes with bounded variables (the components of $\vec{x}(t)$ ), $\left\langle\frac{d \vec{x}(t)}{d t}>=\overrightarrow{0}\right.$. Then we obtain $<\vec{x}(t)>$ by averaging the first equation of $(8)$ :

$$
\langle\vec{x}(t)\rangle=-[A]^{-1}[B]\langle\vec{u}(t)\rangle .
$$

It is interesting to note that in order for $\langle\vec{x}(t)\rangle$ to exist it is sufficient to require boundedness of the components of $\vec{x}(t)$, because from this boundedness the equality $<\frac{d \vec{x}(t)}{d t}>=\overrightarrow{0}$ simply follows. This observation becomes even more essential in the case of an LTV system (e.g. [9]) described by the equations

$$
\begin{aligned}
& \frac{d \vec{x}}{d t}=[A(t)] \vec{x}(t)+[B(t)] \vec{u}(t) \\
& \vec{y}(t)=[C(t)] \vec{x}(t)+[D(t)] \vec{u}(t)
\end{aligned}
$$

For the same assumption of $<\frac{d \vec{x}(t)}{d t}>=\overrightarrow{0}$, we obtain from the first equation

$$
\langle[A(t)] \vec{x}(t)\rangle=-\langle[B(t)] \vec{u}(t)\rangle
$$

which is an integral condition for $\vec{x}$ that can be used for checking any approximation to this vector-function, or be a constraint to a variational method of finding $\vec{x}$. 
Returning to the LTI case, let us illustrate it the with an RLC (resistors, inductors, capacitors) circuit example. Figure 2 shows a scalar (one port and one input) circuit. Here, $v_{i n}(t)$ is the input function and $v_{\text {out }}(t)$ is the output function. It is immediately seen that $H(0)=1 / 3$, that is, $v_{\text {out }}(\infty)=(1 / 3) v_{\text {in }}(\infty)$ if $v_{\text {in }}(\infty)$ exists, and, more generally, $\left\langle v_{\text {out }}(t)\right\rangle=(1 / 3)<v_{\text {in }}(t)>$, regardless of what are the values of the capacitances and inductances.

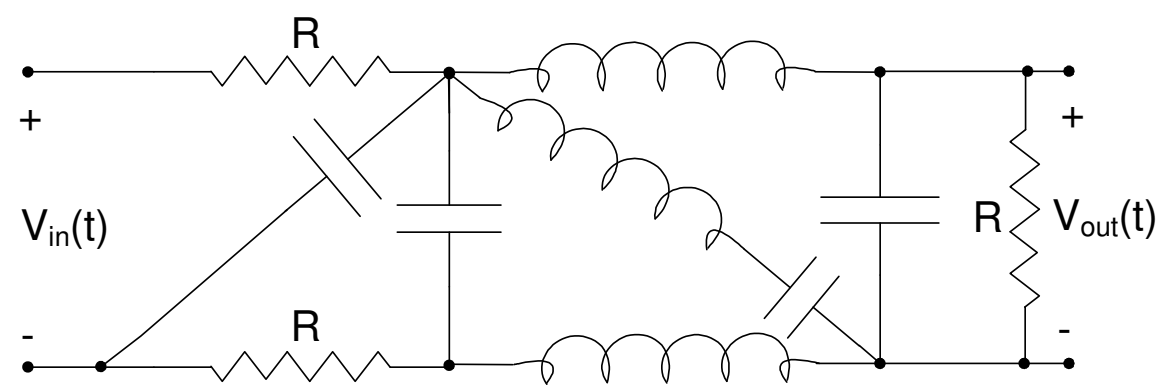

Fig. 2: By disconnecting the capacitors and short-circuiting the inductors, which corresponds to the values of the impedances $s L$ and $(s C)^{-1}$ at $s=0$, we obtain the simple series circuit composed of 3 resistors, each of resistance $R$. Obviously $H(0)=1 / 3$.

\section{Irrational functions}

We turn now to the two main suggestions of [10]: to consider the cases of infinite $f(\infty)$ and irrational functions. The first suggestion is supported by the fact that a solution of a differential equation, linear or not, often has an infinite final value. An example is the queueing process, considered in Section 4, which is unstable, and tends to infinity. However, the suggestion to use irrational functions is more constructive for physical applications, and we focus on it in this section.

\subsection{The irrational degree}

While analyzing the cases when both $f(\infty)$ and $\lim _{s \rightarrow 0} s F(s)$ are infinite, work [10] considers some $f(t)$ for large $t$, employing formulae that are useful for obtaining asymptotic expressions for $f(t)$ as $t \rightarrow \infty$. In particular, it is used that for $\lambda$ not necessarily integer, 


$$
\lim _{t \rightarrow \infty} \frac{f(t)}{t^{\lambda}}=\frac{1}{\Gamma(\lambda+1)} \lim _{s \rightarrow 0} s^{\lambda+1} F(s), \quad \lambda>-1,
$$

where $\Gamma($.$) is the gamma function.$

Equality (9) deserves some analytical commenting. Denoting the common value of the right and left hand sides of (9) as $K(\lambda)$, we have on the one hand

$$
f(t) \sim K(\lambda) t^{\lambda}, \quad t \rightarrow \infty
$$

and, on the other hand,

$$
F(s) \sim K(\lambda) \frac{\Gamma(\lambda+1)}{s^{\lambda+1}}, \quad s \rightarrow 0 .
$$

We may redefine $f$ and $F$ by dividing them both by $K(\lambda)$, and keeping the same functional notations, we obtain the following result.

Theorem 1: Let $F(s)$ be the Laplace transform of $f(t)$. Then, in terms of the asymptotic features of these functions we have the equivalence (" $\Leftrightarrow$ ")

$$
\left(f(t) \sim t^{\lambda}, \quad t \rightarrow \infty\right) \Leftrightarrow\left(F(s) \sim \frac{\Gamma(\lambda+1)}{s^{\lambda+1}}, \quad s \rightarrow 0\right) .
$$

We stress that we speak here only about the asymptotic parts of functions that need not be precisely equal to $t^{\lambda}$ or $\frac{\Gamma(\lambda+1)}{s^{\lambda+1}}$ for all $t$ and $s$ respectively.

Below, we need the following result.

Theorem 2: If for the ratio of the Laplace Transforms $F(s)$ and $G(s)$

$$
\frac{F(s)}{G(s)} \sim s^{-\lambda}
$$

with a non-integer $\lambda$, then at least one of the respective originals, $f(t)$ and $g(t)$, is asymptotically irrational. 
Proof: Assume that both $f(t)$ and $g(t)$ are rational. Then, as is known from the theory of Laplace Transforms, the functions $F(s), G(s)$, and thus their ratio, are rational, which contradicts (11).

The question remains whether or not irrational functions, which cannot be introduced by the use of simple LTI circuits, are practically significant. We therefore now observe how such functions can appear in the analysis of physical systems.

\subsection{The degrees characterizing the irrationality}

Assume that $f(t)$ is some meaningful physical variable related to the description of a medium, and thus this variable is bounded. Then, the time average of $\mathrm{d} f / \mathrm{d} t$, which usually appears in the dynamic description of the medium, is zero. Indeed, since $f(t)$ is bounded,

$$
<\frac{d f}{d t}>=\lim _{t \rightarrow \infty} \frac{1}{t} \int_{0}^{t} \frac{d f(\mu)}{d \mu} d \mu=\lim _{t \rightarrow \infty} \frac{1}{t} \int_{0}^{t} d f(\mu)=\lim _{t \rightarrow \infty} \frac{f(t)-f(0)}{t}=0
$$

The boundedness of $f(t)$ is even an excessive requirement for (12), since for, say, $f(t) \sim t^{1 / 2}(12)$ is still correct. For instance, for a random walk type of process, such that each step takes the same time, we have for the distance $\boldsymbol{R}_{n}$ from the origin after $n$ steps that $\left|\boldsymbol{R}_{n}\right| \sim \sqrt{ }_{n}$ and thus, according to (12), $\left\langle\boldsymbol{R}_{n+1}-\boldsymbol{R}_{n}\right\rangle=\mathbf{0}$, with the average taken over all natural $n$. Thus, (12) leads to physically interesting conclusions.

Next, assume that $\mathrm{d} f / \mathrm{d} t$ is also bounded, which is realistic for many physical processes. The GFVT can then be used on $\langle d f / d t>$ in (12), which leads to

$$
\lim _{s \rightarrow 0} s^{2} F(s)=0
$$

This formula allows us to set some assumptions regarding the asymptotic behavior of $F(s)$ as $s \rightarrow 0$ or, equivalently, regarding the asymptotic behavior of $f(t)$ as $t \rightarrow \infty$, for such realistic processes. Let us consider, for simplicity, a power-law dependence:

$$
F(s) \sim s^{-\alpha}
$$


Then (13) requires $\alpha<2$. For instance, $F(s) \sim s^{-3 / 2}$ is permitted, which means, according to (10), that $f \sim t^{1 / 2}$ as $t \rightarrow \infty$. We note that for $1<\alpha<2$ we obtain $\lim _{s \rightarrow 0} s F(s)=\infty$, that is, an infinite $\langle f\rangle$, which is relevant to the discussion in $s \rightarrow 0$

[10], and for $\alpha<1$, we have $<f>$ finite which is relevant to the discussion in [6].

The hypothesis that we put here forward is that, in many physical problems, the only requirement a priori is the boundedness of $f$ and $\mathrm{d} f / \mathrm{dt}$. This hypothesis would allow us to consider many problems with irrational $f(t)$. However, since the rational case of $F(s) \sim s^{-1}$ is still included, this hypothesis is too general for a decision regarding existence of irrational functions in reality. We thus approach, in the sequel of this section, appearance of irrationality more constructively, stressing the physical side in parallel to the purely mathematical, more straightforward (less restricting $\lambda$ ) reasoning.

\subsection{Non-integer integration and differentiation}

Non-integer integration and differentiation [14-18] is a source of irrational functions. Let us start by recalling some necessary basics. For $n \in \square$, the formula

$$
\int_{0}^{t} d \mu_{1} \int_{0}^{\mu_{1}} d \mu_{2} \ldots \int_{0}^{\mu_{n-1}} f\left(\mu_{n}\right) d \mu_{n}=\frac{1}{(n-1) !} \int_{0}^{t}(t-\mu)^{n-1} f(\mu) d \mu
$$

illustrates the important fact that the solution of an LTI equation can be presented as a convolution. Indeed, the $n^{\text {th }}$-order derivative of each side equals $f(t)$, or, alternatively explained, since $1 / s^{n}$ is the Laplace transform of $t^{n-1} /(n-1)$ !, the transform of each side of (14) is $\left(1 / s^{n}\right) F(s)$. Thus, the $n^{t h}$-order integration of $f(t)$, that is the solution $x(t)$ of the differential equation $\frac{d^{n} x}{d t^{n}}=f(t)$ with zero initial conditions, is identical to the first-order integration with the kernel $(t-\mu)^{n-1}$.

By using a non-integer $\lambda(\operatorname{Re} \lambda>0)$ instead of the integer $n$ in the convolution in the right-hand side of (14), and noting that the Laplace transform of $\frac{t^{\lambda-1}}{\Gamma(\lambda)}$ is $\frac{1}{s^{\lambda}}$, the 
integer-order integration in (14) is generalized to a non-integer (fractional) $\lambda^{\text {th }}$-order integration

$$
D^{-\lambda} f(t)=\frac{1}{\Gamma(\lambda)} \int_{0}^{t}(t-\mu)^{\lambda-1} f(\mu) d \mu
$$

Next, the non-integer differentiation $D^{\lambda} f(t)$ is introduced as the inverse operation.

As an example, consider the non-integer differential equation

$$
\frac{d^{1 / 2} x}{d t^{1 / 2}}=t
$$

According to (14a) we have

$$
x(t)=\frac{1}{\Gamma(1 / 2)} \int_{0}^{t} \frac{\mu}{\sqrt{t-\mu}} d \mu=\frac{1}{\sqrt{\pi}} \int_{0}^{t} \frac{t-\mu}{\sqrt{\mu}} d \mu=\frac{4}{3 \sqrt{\pi}} t^{3 / 2}
$$

The obtained relation $x(t) \sim t^{3 / 2}$ might be expected from the consideration of the physical dimensions, since $\left(d^{1 / 2} x\right) /\left(d^{1 / 2} t\right)=t$ can be rewritten for some small finite differences as $(\Delta x) /\left(\Delta^{1 / 2} t\right)=t$, that is, $\Delta x=t(\Delta t)^{1 / 2}$ which has the dimension of $t^{3 / 2}$.

More generally, the integral in (14a) has the physical dimension of $\left[t^{\lambda}\right][f]$.

Summarizing, integration and differentiation of non-integer order lead to irrational functions. However, the mathematical freedom in the choice of $\lambda$, does not eliminate the engineering importance of ascribing physical sense to these operations. Therefore, the attempt of [17] to use such integration in control theory is important. In what follows, we treat the example of [17], showing that the degree $s^{1 / 2}$ can appear in a physically meaningful 2D problem, described by a linear partial differential equation (PDE), and stress the role of a spatial degree of freedom in this problem.

\subsection{The physical example}

In this example, a semi-infinite cable having distributed (per unit length) resistance $R$ and capacitance $C$ is considered, that is, there are two independent variables, the distance $x$ along the cable, and the time $t$. Contrary to [17], we allow 
not only a voltage but also a current source to be the driver at the beginning of the line, since in modern electronics current sources are commonly met.

The describing equations

$$
\begin{gathered}
-\frac{\partial v}{\partial x}=R i \\
-\frac{\partial i}{\partial x}=C \frac{\partial v}{\partial t}
\end{gathered}
$$

are the distribution versions of the usual equations $v=R i$ and $i=C d v / d t$ for lumped

circuits, while the minuses before $\frac{\partial}{\partial x}$ appear because the positively defined $v(x, t)$ and $i(x, t)$ are decreasing with the distance from the source that is placed at $x=0$.

It is important to observe that $(15 \mathrm{a}, \mathrm{b})$ yield a diffusion (or heat propagation) type of equation for $i(x, t)$,

$$
\frac{\partial^{2} i}{\partial x^{2}}=R C \frac{\partial i}{\partial t}
$$

which means that the mathematical features of the process in the cable are typical for a diffusion process. Diffusion-type processes are a wide class of natural processes, and in some problems, for instance the present one and those associated with radiation, we can speak about the diffusion of energy only. As the point, diffusiontype processes help us to observe the different problems where irrational functions can appear.

Before going further, we note that $R$ and $C$ are spatially distributed parameters and therefore, the expression $R C x^{2}$ (and not $R C$ as for a lumped circuit) is measured in seconds. Thus $R C x^{2} / t$ is a non-dimensional variable in terms of which the similarity of the cable problem to different diffusion systems or processes can be considered.

\subsubsection{The unusual input impedance}

Performing Laplace transforms with respect to $t$ of $(15 \mathrm{a}, \mathrm{b})$, we obtain for the transforms $V(x, s)$ and $I(x, s)$ the equations 


$$
-\frac{d I}{d x}=C s V \quad-\frac{d V}{d x}=R I
$$

Dividing the left equation by the right one, we obtain

$$
\frac{d I}{d V}=\frac{s C}{R} \frac{V}{I}
$$

from which (assuming that $V$ and $I$ can be zero only simultaneously)

$$
I^{2}=\frac{s C}{R} V^{2}
$$

that is

$$
I \sim \sqrt{s} V
$$

The current thus appears to be a derivative of order $1 / 2$ of the voltage. According to Theorem 2, such a situation must yield an irrational dependence on time in at least one of the functions $i(x, t)$ and $v(x, t)$.

Relation (17) is noted in [17], but [17], [18], and some other works considering the semi-infinite cable do not mention the similarity of the electrical process in this system with diffusion-type phenomena. We emphasis the observation that the reason for the irrationality is the additional (spatial) degree of freedom.

The solution of (16) is given by the convolution [19]

$$
i(x, t)=i(0, t) * \frac{(R C)^{1 / 2} x}{2 \sqrt{\pi} t^{3 / 2}} e^{-\frac{R C x^{2}}{4 t}}
$$

where $i(0, t)$ is the input current source, $i_{s}(t)$. Having the form of a zero-state response, (18) corresponds to the case when the source is connected at $t=0$ to the line without any current; i.e. $i(x, 0) \equiv 0$. Observe also that the unit of ampere is obtained because the integration by $t$ in the convolution adds second to the dimension (while $(R C / t)^{1 / 2} x=\left(R C x^{2} / t\right)^{1 / 2}$ is non-dimensional). 
As the main point of this example, for any rational source-function $i(0, t)$, the solution function $i(x, t)$ is irrational. This is the feature of the cable as a diffusive system. For an input voltage source $v_{s}(t)=v(0, t)$, the problem of the semi-infinite cable is solved similarly. (See also [17].)

\subsubsection{Comparison with other physical systems}

That $V / I \sim s^{-1 / 2}$ in (17), means that the input impedance of the semi-infinite cable in terms of the frequency response equals $Z(\omega) \sim \omega^{-1 / 2}$. It is interesting to note, for comparison, that a lumped inductor $L$ having an iron core composed of insufficiently thin layers, has $Z_{L}(\omega) \sim \omega^{1 / 2}$. The latter is because the depth of penetration (or decay) of electromagnetic field into iron (the known skin effect) is proportional to $\omega^{-1 / 2}$. Since the thick layers are not completely filled by the magnetic field, the inductance is proportional to the depth of the penetration, and thus is frequency-dependent; which yields $Z_{L}(\omega)=j \omega L \sim \omega \omega^{-1 / 2}=\omega^{1 / 2}$ (more precisely, $\left|Z_{L}(\omega)\right| \sim \omega^{1 / 2}$, because for such inductor $\left.\operatorname{Re}\left[Z_{L}(\omega)\right] \approx \operatorname{Im}\left[Z_{L}(\omega)\right]\right)$, instead of $Z_{L}(\omega) \sim \omega$ for the usual inductor. Note

that the decaying of electromagnetic field in any conductive medium (here of the inductor's core) is also a diffusion-type process.

Some works in which irrationality of the type $s^{1 / 2}$ is obtained in other physical systems, are cited in [18].

The role of the spatial degree(s) of freedom, observed in the diffusion equation, is also well seen in the problem of Brownian movement; more generally, in the "1/fnoise" systems problems. In such problems, the power spectrum of a time function, i.e. the square of $F(j \omega)$, or $F(s)$, is observed to be proportional to $1 / s$, which means that $F(s)$ is proportional to $s^{-1 / 2}$. The time processes in such systems are associated with the spatial movement of some particles; the processes in these additional degrees of freedom cause the irrationality in the time-dependence.

A relevant physical outlook on the possible irrationality of the solution of such a spatially-temporally is obtained when we consider the role of time inversion, as follows. 


\subsubsection{The degree of irrationality and time-inversion}

In the field of PDE, irrational functions are obtained not only for the diffusion-type equation, but also for the wave equation with a dissipation (friction) term. Consider

$$
\frac{\partial^{2} u}{\partial t^{2}}=a^{2} \frac{\partial^{2} u}{\partial x^{2}}+b \frac{\partial u}{\partial t}
$$

where $a$ and $b$ are positive constants. Work [19] treats this equation by describing the oscillations of a string with a damping given by $b$. It is shown in [19] that for any fixed $x$, the oscillations of the string, caused by a pinch, are decaying as $t^{-1 / 2}$. The irrationality can be explained by the role of the terms in (19) and (16), which include $\frac{\partial}{\partial t}$, responsible for the dissipation of energy. Such a term is associated with the irreversibility of the dissipation process; reversibility would contradict the fact of the heating that causes the damping, that is, the law of increase of entropy. Contrary to that, the undamped wave equation $(b=0)$ is not changed by $t \rightarrow-t$, and the undamped wave would propagate as it is with the time inversion in the back direction, which means reversibility of the process.

It is most interesting to observe, however, how simply the mathematics "formalizes" the fact of irreversibility of the process described by such equation. Since such factors as $t^{ \pm 1 / 2}$ or $t^{ \pm 3 / 2}$, appearing in the solution functions, include $\sqrt{ } t$, the physical requirement of realness of the solution function quite formally prohibits negative $t$, that is, time inversion. For this reason, we can also expect, for instance, such a degree as $t^{3 / 4}$, but not as $t^{1 / 3}$ or $t^{3 / 5}$, to arise in physics problems with irreversibility: in statistical theories, hydrodynamics, and non-stationary problems of quantum mechanics.

The above arguments suggest, furthermore, to consider PDE-s having a term which is not necessarily diffusive, but which reverses its sign with inversion of time, for instance $t \frac{\partial^{2} u}{\partial t^{2}}$, or $t^{3} \frac{\partial^{2} u}{\partial x^{2}}$. 


\subsection{Ordinary equations with singular coefficients}

For our last, and again purely mathematical, example of obtaining irrational functions, we return to ordinary (1D) differential equations, again dealing with functions of only time.

It is known from the analytical theory of ordinary (generally, complex) differential equations [19] that, if the coefficients in the equations are singular functions, the solution function can include non-integer degrees. For instance, seeking solution of the equation

$$
\frac{d^{2} f}{d t^{2}}+\frac{a}{t} \frac{d f}{d t}+\frac{b}{t^{2}} f=0,
$$

with $a$ and $b$ constants, of the form $t^{\lambda}$, we obtain for $\lambda$ the characteristic equation $\lambda^{2}+(a-1) \lambda+b=0$. The roots $\lambda_{1}$ and $\lambda_{2}$ (needed to form $f(t)=A t^{\lambda_{1}}+B t^{\lambda_{2}}$, with $A$ and $B$ constants) need not be integers. Thus, ordinary differential equations including derivatives of integer order, but having singular coefficients also can be a source of irrational functions.

We conclude that realization of irrational functions is an interesting research direction.

\subsection{Some further comments on [10]}

1. Being concerned with composition of functions, one notes that the usual partial fraction expansion of $F(s)$, appearing in the theory of the LTI circuits [7,8], is a composition of $f(t)$ using some linearly independent functions. The rational $\{F(s)\}$ form a (mathematical) field [20] since summation and multiplication operations, and the inverse operations subtraction and division, always preserve the rational structure. Contrary to this, irrational functions do not form any linear space, since the terms responsible for irrationality can be mutually canceled with additions or subtractions. The irrational functions are treated in [10] as some individual objects, and investigation of how such functions can compose each other, that is, how the sets of such functions can be used effectively for particular problems in control theory, should also be a research continuation on the line of [10]. 
2. Circuit specialists use the frequency response $H(j \omega)(H(s), s \rightarrow j \omega$, $\omega \in \mathbf{R}$ ) only for systems possessing a finite and stable impulse response $h(t)$, when $h(\infty)$ exists and is finite. Thus, the cases when $f(t)$ is the impulse response of a system have to be carefully separately considered as regarding application of the conclusions of [10] related to $f(t) \rightarrow \infty$.

3. Results of [10] relate only to continuous functions and should be generalized to the important discrete case, that is, to sequences and their z-transforms. In the section 4 , we encounter a discrete sequence $f_{n} \rightarrow \infty$, with fractional degree.

\section{Time average instead of final value}

In this last section, we present some elements relevant to the use of the GFVT. We provide some proofs, discuss the discrete case and go into some applications.

\subsection{Proofs}

\subsubsection{Periodic functions}

Following [6], let us first prove the GFVT (equation (2)) for periodic functions that (when not constant) have no $f(\infty)$, using the known formula for the Laplace transform of a $T$-periodic function, $f(t+T)=f(t)$,

$$
\begin{aligned}
& s F(s)=s \frac{\int^{T} e^{-s t} f(t) d t}{1-e^{-s T}} \underset{s \rightarrow 0}{\rightarrow} \frac{1}{T} \int_{0}^{T} f(t) d t \\
& =\lim _{t \rightarrow \infty} \frac{1}{t} \int_{0}^{t} f(t) d t
\end{aligned}
$$

At the last step of this derivation, we used that for a $T$-periodic function $\langle f(t)\rangle_{T}=$ $<f(t)>$.

It is obvious from (20) that, for any periodic function, $\lim _{s \rightarrow 0} s F(s)$ and $<f>$ exist simultaneously. 


\subsubsection{On the spectral composition of $f(t)$}

An alternative way to obtain (20) is given by employing a constant function together with sinusoidal functions, and using the Fourier expansion of any periodic function. This possibility is more far-reaching since then the use of the linearity of the operator $s F(s)$ with regard to $F($.$) and the approaching F($.$) as a composition,$ allows us to consider any real-valued almost periodic function

$$
f(t)=\sum_{k} c_{k} e^{\lambda_{k} t}
$$

with any $\left\{\lambda_{k}\right\}$ (when complex, in conjugated pairs). For instance, $A+B \sin \omega t+C \sin \sqrt{2} \omega t$ is also included. Such functions can be relevant to different stochastic-processes applications, as those in [1-5].

It is seen from the above construction that when spectral analysis of an $f(t)$ (not necessarily periodic) is possible, then $\lim _{s \rightarrow 0} s F(s)$ and $\langle f(t)>$ exist simultaneously, and for such functions the GFVT is also proved.

\subsubsection{Comments}

1. While for the case of $f(\infty)$ existing (and then also $\left.f^{2}(t) \rightarrow f(\infty)^{2}\right)$, we have for the dispersion of $f(t)$

$$
\begin{aligned}
& \left\langle(f-<f>)^{2}\right\rangle \equiv<f^{2}>-<f>^{2}, \\
& =f(\infty)^{2}-f(\infty)^{2}=0
\end{aligned}
$$

for $f(\infty)$ not existing, any non-constant bounded asymptotic form of $f(t)$, for instance that given by equation of the type (21), always provides a nonzero dispersion.

2. For a uniformly limited (not tending to infinity) $f(t)$, the non-existence of $f(\infty)$ always means some oscillations of $f(t)$.

3. In many physical systems, large-scale time processes are not stabilized, and we can speak just about $\langle f(t)>$. An example can be, for instance, intensity of wind velocity at a certain spatial point in the atmosphere. This parameter can be important, for wind stations generating electrical energy, where the pressure and the energetic 
action are defined by square of the velocity function $v(t)$, and the Laplace transform of $v^{2}(t)$ should be considered.

\subsection{The discrete case}

Using arguments, similar to those that led to (2), work [6] also suggests the discrete analog of (2) for the z-transform $F(\mathrm{z})$ of a sequence $\left\{f_{n}, n \geq 0\right\}$,

$$
F(z)=\sum_{0}^{\infty} f_{n} z^{-n}
$$

namely

$$
\lim _{z \rightarrow 1+}(1-z) F(z)=\left\langle f_{n}\right\rangle
$$

which generalizes the classical discrete final value theorem [3]

$$
\lim _{z \rightarrow 1+}(1-z) F(z)=f_{\infty}
$$

Thus, sequences $f_{n}$ not having a limit $f_{\infty}$ can be considered. When $f_{\infty}$ exists, $\left\langle f_{n}\right\rangle=f_{\infty}$, i.e., (1a) is included in (2a), as is the case for continuous functions.

However, many sequences have average value $\left\langle f_{n}\right\rangle$, but not a final value $f_{\infty}$. For instance, the sequence $\{1,-1,1,-1, .$.$\} has a zero average but no limit value, and the$ same goes for $\{1,0,0,1,0,0,1, \ldots\}$ with $\left\langle f_{n}\right\rangle=1 / 3$. All these cases fit (2a).

\subsection{Applications of the GFVT}

Quoting [6], works [1,2] apply (2a) to the theory of queueing (in particular in the Internet), and works [3-5] apply (2) to the theory of Brownian motion. In all these works, long-term time processes are considered.

\subsubsection{Brownian motion}

The motivation in [3-5] is associated with ergodicity of some physical systems, that is, with the equality of statistical (ensemble) averages and time averages over the trajectory of a single particle. Allowing calculation of a time average of a variable 
over a trajectory, the GFVT gives the statement of ergodicity a constructive meaning. Namely, a time function, for which the time average is treated as in [6], is associated in [3-5] with such a trajectory, and then the ergodicity helps in calculating thermodynamic properties of a physical medium, which, by definition, are based on the ensemble averages. We will not go here into further details about this application of the GFVT.

\subsubsection{Use of the GFVT in queueing theory}

The application of the GFVT in $[1,2]$ relates to a queueing problem in the Internet. Since this application is relevant also to [10], it is therefore chosen to be considered in more detail.

Queueing theory is the mathematical study of queueing phenomena which occur in many daily applications. Modern applications of queueing theory are the design and operation of telecommunication and computer networks, such as the Internet.

In abstract terms, a queueing system is composed of one or more service units and a queue. The service units perform some kind of service to customers, whereas the queue is used by customers to wait until a service unit can service them. Customers arrive in the queueing system, wait in the queue until a service unit becomes available, are served by one of the service units, and leave the queueing system (see Figure 3).

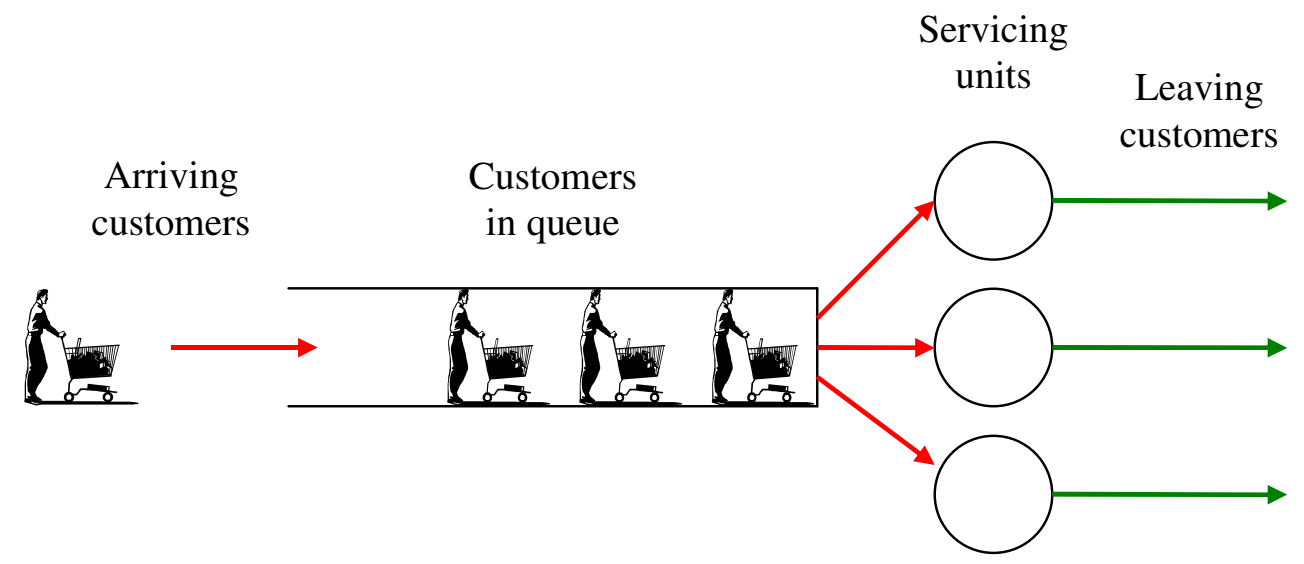

Fig. 3: A queueing system consists of servicing units and a queue. Customers require the service of the servicing units and go through 4 consecutive stages: they arrive in the queueing 
system, wait in the queue until one of the servicing units becomes available, are served by this service unit and finally leave the queueing system.

In the study of a particular queueing system, three steps are distinguished. First, the queueing system is transformed to a mathematical queueing model. This step encompasses modeling of the arrival and service process. The arrival process describes the way in which customers arrive to the queueing system, while the service process summarizes the way in which customers are serviced. It is important to note that we assume a stochastic framework. In general, all the variables are stochastic. Second, we determine the performance measures we want to calculate. Popular performance measures are the expected values of the number of customers in the system and of the sojourn time of a customer. Third, the queueing model is analyzed.

The transform technique is one of the most popular analysis techniques. With this technique, the equations that relate the output to the input are transformed to the Laplace and/or $z$-transform domain, and solved in this domain. Finally, performance measures are calculated from the transforms.

To clarify the use of the FVT and GFVT in queueing theory, let us consider an example taken from [1], introducing first the mathematical terminology.

\subsubsection{Some basic concepts for the modeling}

In the queueing model, time is discretized. It is divided into slots, all of equal length. The number of customer arrivals, $e_{k}$, in the $k$-th slot is independent of the numbers of customer arrivals in other slots. The $e_{k}(k \geq 1)$ have a common probability generating function (PGF) $E(z)$, defined as $\mathrm{E}\left[z_{k}^{e}\right]$, with $\mathrm{E}[$.$] the expected value$ operator of a stochastic variable. Note that $E(z)$ characterizes the arrival process completely. We further assume one service unit that services all the customers in a First-In-First-Out manner. When customers are present in the system at the beginning of a certain slot, the service unit starts to service the customer that is waiting the longest in the queue. This service ends at the end of the slot. As a final input, we characterize the sojourn time of the first arriving customer. A natural assumption is that the first customer arrives in a system with no customers present. He can then be served directly and his sojourn time is equal to exactly one slot. However, in [1] we 
allow for a more general sojourn time of the first arriving customer. We merely assume that its PGF, $D_{1}(z)$, is given.

The performance measure of interest is the expected sojourn time, $\mathrm{E}\left[d_{n}\right]$, of the $n$-th arriving customer in the system. More precisely, we want to know the number of slots the $n$-th arriving customer is expected to be present in the queueing system. The expected sojourn times can be regarded as a sequence $\left\{f_{n}, n \geq 1\right\}$, with $f_{n}=\mathrm{E}\left[d_{n}\right]$. Since the calculation of the complete sequence $\left\{f_{n}, n \geq 1\right\}$ is not straightforward, calculation of $f_{\infty}$ (if it exists) or of $\left\langle f_{n}>\right.$ is of great interest. This is where the (G)FVT comes into play.

\subsubsection{Thus the mathematics works}

In order to calculate the average expected sojourn time $<f_{n}>$, we start from the $z$-transform of the sequence $\left\{f_{n}, n \geq 1\right\}$. It is obtained in [1] as

$$
F(z)=\frac{f_{1} z}{1-z}+\frac{z^{2}}{(1-z)^{2}}+\frac{(E(z)-E(0)) z}{(1-E(0))(1-z)(E(z)-1)}+\frac{(Y(z)-E(0)) z D_{1}(Y(z))}{(1-E(0))(1-z) Y(z)(1-Y(z))},
$$

with $f_{1}$ the expected sojourn time of the first customer, $E(z)$ the PGF of the number of arrivals in a slot, $D_{1}(z)$ the PGF of the sojourn time of the first customer, and $Y(z)$ the unique solution of the equation $x-E(x z)=0$, inside the complex unit disk of the $x$ plane for all $z,|z|<1$. Here, $D_{1}(z), f_{1}$ and $E(z)$ are input functions and $F(z)$ is the output function. Using the GFVT [6] to the above expression for $F(z)$, we can calculate $\left\langle f_{n}\right\rangle$ explicitly as

$$
<f_{n}>=\lim _{z \longrightarrow 1}(1-z) F(z)=\left\{\begin{array}{c}
1+\frac{E^{\prime \prime}(1)}{2 E^{\prime}(1)\left(1-E^{\prime}(1)\right)} \text { if } E^{\prime}(1)<1 . \\
\infty \text { if } \quad E^{\prime}(1) \geq 1
\end{array}\right.
$$

Here, $E^{\prime}(1)$ and $E^{\prime}$ (1) are the first and second derivative of $E(z)$ at $\mathrm{z}=1$. Note that l'Hopital's rule is used multiple times in the case $E^{\prime}(1)<1$, since $E(1)=Y(1)=1$, by definition. Equation (22) shows that if $E^{\prime}(1)<1$ and $f_{\infty}$ exists, the above formula yields the final value $f_{\infty}$ of the sequence $\left\{f_{n}, n \geq 1\right\}$. 
In queueing-theoretic terms, $f_{\infty}$ is called the steady-state value, or the expected sojourn time when the system has reached a steady state. This value can be found by the standard FVT. However, the GFVT is more generally applicable as it yields the average expected sojourn time if $E^{\prime}(1)<1$ also for $f_{\infty}$ not existing [6]. Furthermore, it correctly shows the tendency to infinity if $E^{\prime}(1) \geq 1$, which is relevant to [10]. In the latter case, we have an unstable queueing system. This can be understood as follows: on average more customers arrive in the system than can be serviced. The number of customers waiting in the queue therefore increases unboundedly in time. The expected sojourn time of customers is also increasing and $f_{n} \rightarrow \infty$ for $n \rightarrow \infty$. Some illustrating examples for the three distinguished cases are shown in Figure 4.

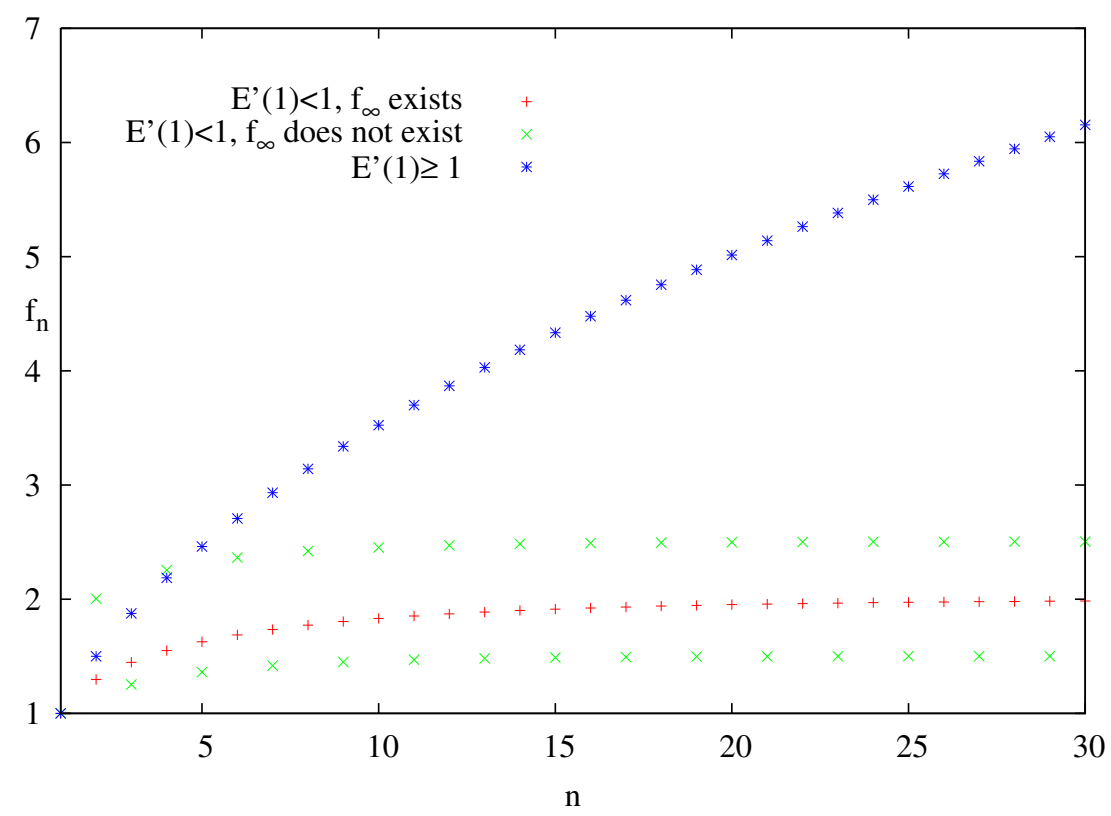

Fig. 4: Three illustrative examples of the sequence $\left\{f_{n}, n \geq 1\right\}$, with $f_{n}$ the expected sojourn time of the $n$-th arriving customer in the queueing system. The red sequence has a final value $f_{\infty}$ equal to 2 . The green "two-level" sequence does not converge to a final value, but its average $\left\langle f_{n}>\right.$ equals 2 as well. The blue sequence is unbounded and thus $f_{n} \rightarrow \infty$ for $n \rightarrow \infty$. More precisely, $f_{n} \sim n^{1 / 2}$ in this case. The red sequence can be treated with the standard FVT; the green sequence is an example where the GFVT comes in handy, while the blue sequence is related to the irrational functions treated in [10]. 


\section{Conclusions}

Two generalizations of the final value theorem $[6,10]$ were considered. In both cases, the final value theorem is extended to cases when the final value of the function of interest does not exist. Both extensions trigger some interesting research questions, which we have touched upon in this paper. For the line of [10], we have suggested the consideration of compositions of irrational functions, the exploration of the physical origination of these functions, and the extension to the discrete case. For the line of [6], we have focused on the role of transfer functions and on applications in the fields of classical and stochastic control. The whole field of possible applications of the GFVT is shown to be interesting and promising, especially for stochastic problems.

We hope to have given the reader a taste of possible future research directions originating from the treated generalizations of the final value theorem.

Acknowledgments: Professor Menachem Krichevsky gave us the example of the frequency dependent inductor (Section 3.4.2). The Fund for Scientific Research, Flanders (F.W.O.-Vlaanderen), Belgium, supports Joris Walraevens as its Postdoctoral Fellow.

\section{References}

[1] Walraevens, J., Fiems, D. and Moeneclaey, M. (2009) 'Using singularity analysis to approximate transient characteristics in queueing systems', Probability in the Engineering and Informational Sciences, 23(2), 333-355.

[2] Walraevens, J., Fiems, D., Moeneclaey, M., and Bruneel, H. (2007), 'A generic approximate transient analysis applied to a priority queue', Proceedings of the 3rd EURONGI Conference on Next Generation Internet Networks (NGI 2007), 28-35.

[3] Lapas, L.C., Morgado, R., Vainstein, M.H., Rubi, J.M., and Oliveira, F.A. (2008), 'Khinchin theorem and anomalous diffusion', Phys. Rev. Lett., 101, 230602.

[4] Lapas, L.C. (2008), 'Difusao anomala e termodinamica de nanoparticulas', PhD thesis in Physics, Universidade de Brasília, Instituto de Física. 
[5] G.R. Rezende, L.C. Lapas, F.A. Oliveira, "Complexity perspectives: an anomalous diffusion approach", Journal of Computational Interdisciplinary Sciences 1 (2), 105-111 (2009).

[6] Gluskin, E. (2003), 'Let us teach this generalization of the final-value theorem', European J. of Physics, 24(6), 591-597.

[7] Doetsch, G. (1974), Introduction to the Theory and Application of the Laplace Transformation, Berlin, Springer-Verlag.

[8] Hayt, W.H., and Kemmerly, J.E. (1993), Engineering circuit analysis (Fifth edition), McGraw-Hill, New York.

[9] Zadeh, L.A., and Desoer, C.A. (1963), Linear System Theory, McGraw-Hill, New York.

[10] Chen, J., Lundberg, K.H., Davison, D.E., and Bernstein, D.S. (2007), 'The final value theorem revisited (infinite limits and irrational functions)', IEEE Control Systems Magazine, 27(3), 97-99.

[11] Hamming, R.W. (1962), Numerical methods for Scientists and Engineers, McGraw-Hill, New York.

[12] Gluskin, E. (2004), 'Spatial filtering through elementary examples', European J. of Physics, 25(3), 419-428.

[13] Gluskin, E. (2005), 'On the ideal low-frequency spatial filtration of the electrical potential at infinity', Physics Letters A, 338(3-5), 209-216.

[14] Courant, R. (1964), Differential and Integral Calculus, vol. II, Interscience/Wiley, New York.

[15] Gel'fand, I. M., and Shilov, G.E. (1964), Generalized Functions. Volume 1: Properties and Operations, Academic Press, New York.

[16] Barnes, J.A., and Allan, D.W. (1966), 'A statistical model of flicker noise', Proceedings of the IEEE, 54(2), 176-178.

[17] Manabe, S. (1961), 'The non-integer integral and its application to control systems', English Translation Journal Jpn., 6(3-4), 83-87

[18] Hartly, T.T., Lorenzo, C.F., and Qammer, H.K. (1995), 'Chaos in a fractional order Chua's system', IEEE Transactions on Circuits and Systems--I: Fundamental Theory and Applications, 42(8), 485-490.

[19] Sidorov, Yu.V., Fedoryuk, M.V., and Shabunin, M.I. (1985), Lectures on the theory of functions of a complex variable, Mir, Moscva.

[20] van der Waerden, B.L. (1955), Algebra Springer, Berlin. 